\section{Pink or blue: why and when to use pulse oximetry}

\section{Sam McMillan}

Hypoxaemia can be defined as low oxygen content in arterial blood. This can be caused by reductions in arterial oxygen tension $\left(\mathrm{PaO}_{2}\right)$, decreases in oxygen haemoglobin saturation $\left(\mathrm{SpO}_{2}\right)$ and decreases in haemoglobin concentration.

Decreases in $\mathrm{PaO}_{2}$ are caused by:

- Low inspired oxygen levels $\left(\mathrm{FiO}_{2}\right)$

- Hypoventilation

- Diffusion barrier (i.e. pulmonary oedema/pus in alveoli)

- Ventilation:perfusion inequality

- Intrapulmonary shunt (causing blood to pass through the lungs without undergoing gaseous exchange).

Decreases in $\mathrm{SpO}_{2}$ are caused by:

- Decreases in $\mathrm{PaO}_{2}$

- The formation of methaemoglobinaemia or carboxyhaemoglobinaemia.

Decreases in haemoglobin concentration are caused by anaemia.

Pulse oximetry can be used to determine the arterial haemoglobin saturation with oxygen $\left(\mathrm{SpO}_{2}\right)$. In a healthy patient the haemoglobin should be $>95 \%$ saturated with oxygen. We can expect the anaesthetized patient breathing $100 \%$ oxygen to have a $\mathrm{SpO}_{2}$ reading closer to $100 \%$. It is a monitor that mainly becomes useful in the recovery stages of anaesthesia and later in intensive care.

The relationship between $\mathrm{PaO}_{2}$ and $\mathrm{SpO}_{2}$ forms a sigmoid curve, meaning that below $93 \% \mathrm{SpO}_{2}$ a small decrease in $\mathrm{SPO}_{2}$ will result in a large decrease in $\mathrm{PaO}_{2}$. Therefore pulse oximeter readings of $93 \%$ or higher are acceptable in non-anaemic critically ill patients. But as $90 \% \mathrm{SpO}_{2}$ correlates to $\mathrm{PaO}_{2}$ of $60-70 \mathrm{mmHg}$ (severe hypoxaemia), oxygen should be supplemented where readings on the pulse oximeter are less than $93 \%$. As a rule of thumb below $90 \% \mathrm{SpO}_{2}, \mathrm{PaO}_{2}$ is approximately $\mathrm{SpO}_{2}$ minus 30. Always try moving the probe to another location before assuming hypoxaemia, as the place where you have located the probe may merely be poorly perfused.

Pulse oximetry uses a simple principle that oxygenated blood is a different colour to blood that is not well oxygenated. Light is passed through a pulsating arterial vascular bed and the pulse oximeter can detect the oxygen saturation within that artery. It disregards absorption from tissues that are not pulsating, that is, venous blood, skin and muscle. Oxyhaemoglobin and deoxyhaemoglobin give different light wavelengths which allow the microprocessor to detect the saturation. Some pulse oximeters may not distinguish dysfunctional haemoglobin such as methaemoglobin or carboxyhaemoglobin.

The probe can be placed on various sites including the tongue, pinna, lip, toe web and tail, but is sometimes not well tolerated in conscious patients. It is however minimally invasive and where arterial blood gases are not available it can be a useful tool in monitoring trends and disease progression in hypoxaemic patients and for tailoring oxygen therapy post anaesthesia.

The major limiting factor of pulse oximetry is tissue perfusion. Conditions such as shock ad hypotension, which reduce peripheral blood flow, will prevent the pulse oximeter from accurately reading haemoglobin saturation. It should be noted that fluorescent lighting, pigmentation, compressed tissue (from leaving the probe in one place for too long), cold extremities and patient movement can all interfere with pulse oximeter readings.

Pulse oximetry is not accurate below an oxygen saturation of $75 \%$.

It should also be noted that the pulse oximeter gives us no indication of the oxygen content of the blood, the amount of oxygen dissolved in the blood $\left(\mathrm{PaO}_{2}\right)$, ventilation, cardiac output or blood pressure.

\section{KEY LEARNING OBJECTIVES}

- Understand the physiology of oxygenation

- Understand how pulse oximetry works

- Understand when and why to use pulse oximetry

\section{MULTIPLE CHOICE QUESTIONS}

1. Pulse oximetry measures which of the following?
(A) Oxygen saturation of haemoglobin
(B) Partial pressure of oxygen dissolved in the blood
(C) End-tidal carbon dioxide
(D) Oxygen perfusion

2. Methaemoglobin may be caused by the ingestion of which ONE of the following?
(A) Ethylene glycol
(B) Smoke inhalation
(C) Theobromine
(D) Paracetamol

3. If an abnormal reading is detected on pulse oximetry, which of the following is the LEAST appropriate action?
(A) Repeatedly move the probe
(B) Assess the pulse rate reading on the monitor with a manual pulse reading
(C) Assess the plethysmographic waveform for clear, visible pulse waveforms
(D) Thoroughly assess the patient 